\title{
Review Article \\ Molecular Design and Applications of Self-Assembling Surfactant-Like Peptides
}

\author{
Chengkang Tang, ${ }^{1,2}$ Feng Qiu, ${ }^{1,2}$ and Xiaojun Zhao ${ }^{1}$ \\ ${ }^{1}$ Nanomedicine Laboratory, West China Hospital, Sichuan University, Chengdu 610041, China \\ ${ }^{2}$ Regenerative Medicine Research Center, West China Hospital, Sichuan University, Chengdu 610041, China \\ Correspondence should be addressed to Xiaojun Zhao; xiaojunz@mit.edu
}

Received 17 July 2013; Accepted 12 August 2013

Academic Editor: Yuan Zhang

Copyright (c) 2013 Chengkang Tang et al. This is an open access article distributed under the Creative Commons Attribution License, which permits unrestricted use, distribution, and reproduction in any medium, provided the original work is properly cited.

Self-assembling surfactant-like peptides have been explored as emerging nanobiomaterials in recent years. These peptides are usually amphiphilic, typically possessing a hydrophobic moiety and a hydrophilic moiety. The structural characteristics can promote many peptide molecules to self-assemble into various nanostructures. Furthermore, properties of peptide molecules such as charge distribution and geometrical shape could also alter the formation of the self-assembling nanostructures. Based on their diverse self-assembling behaviours and nanostructures, self-assembling surfactant-like peptides exhibit great potentials in many fields, including membrane protein stabilization, drug delivery, and tissue engineering. This review mainly focuses on recent advances in studying self-assembling surfactant-like peptides, introducing their designs and the potential applications in nanobiotechnology.

\section{Introduction}

Molecular self-assembly is a universal phenomenon in nature: phospholipid molecules can self-assemble into millimeter-size lipid tubules, silk fibroins can be fabricated into silk materials over $2 \mathrm{~km}$ in length [1], and so on. Molecular self-assembly is usually driven by noncovalent bonds such as ionic bond, hydrophobic interaction, van der Waals interaction, and hydrogen bonding, which could promote self-assembling molecules to spontaneously aggregate into well-ordered structures. As a bottom-up strategy to fabricate nanomaterials, molecular self-assembly has received considerable attentions.

Recently, nature-inspired investigators have designed novel nanobiomaterials based on self-assembling peptides, which are usually composed of natural L-amino acids and have excellent biocompatibility. As a novel category of selfassembling nanomaterials, self-assembling peptides have become especially attractive, for their successful applications in many fields, including three-dimensional cell culture and reparative or regenerative medicine [2-8], tissue engineering [9-11], and drug release [12-14]. In this review, we will focus on a family of surfactant-like peptides designed by mimicking the structure of traditional surfactants and introduce their applications in biological surface engineering.

\section{Design of Typical Surfactant-Like Peptides}

Surfactants are defined as materials that can greatly decrease the surface tension of solvents when used at very low concentrations [15]. Surfactants are usually a category of amphiphiles composed of hydrophobic tail and hydrophilic head. Recently, a family of surfactant-like peptides has been designed by mimicking the structure of traditional surfactants [16-18]. A typical surfactant-like peptide molecule consists of two parts: a hydrophobic tail composed of several consecutive hydrophobic amino acids and a hydrophilic head composed of one or two hydrophilic amino acids.

Based on this rule, researchers have selected different hydrophobic or hydrophilic amino acids to design various surfactant-like peptides freely. For example, the hydrophilic head could be designed as positively charged Arg, Lys, and His, or negatively charged Asp and Glu, producing cationic or anionic surfactant-like peptides. On the other hand, the hydrophobic tail could be designed by choosing different 

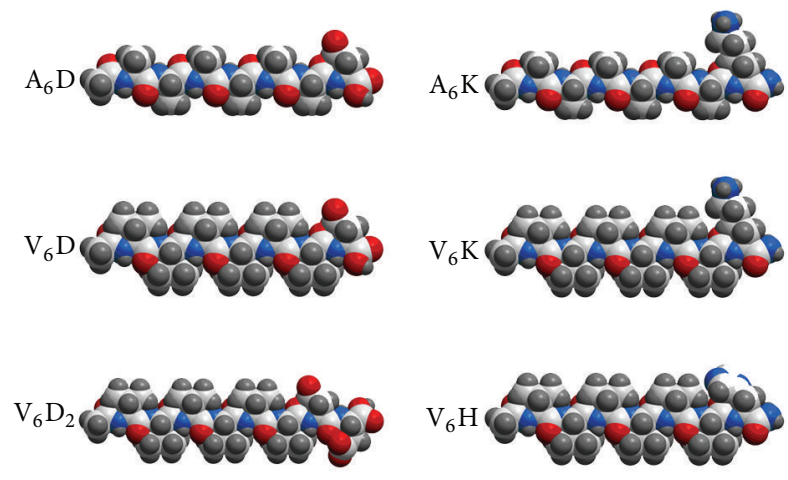

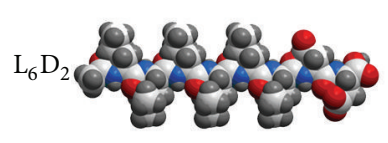

(a)

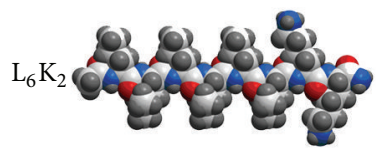

(b)
FIGURE 1: The molecular models of several classic surfactant-like peptides. (a) Negatively charged surfactant-like peptides; (b) positively charged surfactant-like peptides, grey $=$ hydrogen atom, white $=$ carbon atom, red $=$ oxygen atom, blue $=$ nitrogen atom .

hydrophobic amino acids such as Gly, Ala, Val, Leu, and Ile with different levels of hydrophobicity, so that the overall hydrophobicity of a surfactant-like peptide could be controlled. Recently, a number of typical surfactant-like peptides have been designed (Figure 1).

The flexible design of typical surfactant-like peptide is not based on the selection of amino acids only. The position of the hydrophilic head could be changed to generate new surfactant-like peptides. An example is that the hydrophilic heads of those peptides in Figure 1 are set at the C-terminal, while the heads of KVVVVVV $\left(\mathrm{KV}_{6}\right)$ and HHVVVVVV $\left(\mathrm{H}_{2} \mathrm{~V}_{6}\right)$ are set at the $\mathrm{N}$-terminal [18]. The length of a surfactant-like peptide could also be a design strategy by usually controlling the number of hydrophobic amino acids in the tail. Although the tail of a surfactant-like peptide molecule is generally composed of six hydrophobic amino acids, making the whole peptide molecule about $2.5 \mathrm{~nm}$ in length, which is similar to the length of natural phospholipids, novel surfactant-like peptide molecules, with a longer hydrophobic tail, have the same self-assembling behavior, except that the self-assembling nanostructures become more polydisperse [17].

\section{Self-Assembling Mechanism of Typical Surfactant-Like Peptides}

Like the classic formation of lipid bilayer, one self-assembling model of surfactant-like peptides is proposed that molecules could undergo a tail-to-tail alignment to form bilayer structures, which further form nanotubes and nanovesicles (Figure 2(a)). In this model, surfactant-like peptides, such as $\mathrm{A}_{6} \mathrm{D}, \mathrm{V}_{6} \mathrm{D}, \mathrm{V}_{6} \mathrm{D}_{2}, \mathrm{~L}_{6} \mathrm{D}_{2}$, are composed of $1 \sim 2$ charged amino acids and $4 \sim 8$ hydrophobic amino acids and undergo self-assembly in aqueous solution to form nanotubes or

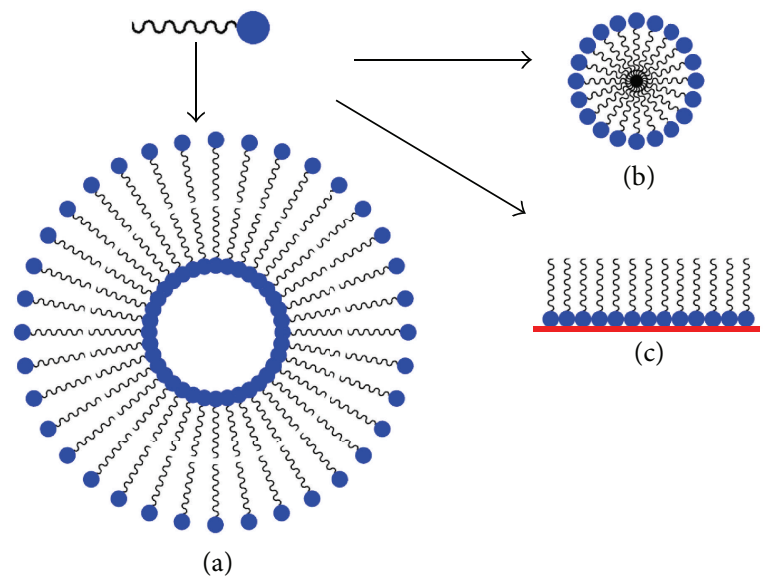

FIgure 2: The self-assembling models of typical surfactant-like peptides. (a) Bilayer tube or vesicle. (b) The formation of micelle. (c) Monolayer on mica surface.

nanovesicles with diameter of $30 \sim 50 \mathrm{~nm}$, which could further form network with three-way junctions [16-20]. These nanostructures were highly unstable and dynamic so that a special quick-freeze/deep-etch technique was required to fix and visualize them. These peptide molecules take an irregular secondary structure as revealed by circular dichroism (CD), which seems less important for the self-assembling process, and the hydrophobic interaction between tails is regarded as the major driving force. Generally, surfactant-like peptides with tail of Ala or Val could form more stable nanostructures than those with tail of Gly, Leu, and Ile $[15,16]$.

In addition to forming tail-to-tail bilayer structure, traditional surfactants could also form micelles by packing the tails in a hydrophobic core and exposing the hydrophilic heads outside. This kind of self-assembling model has also been observed for surfactant-like peptides, which could form nanofibers instead of nanotubes or nanovesicles [21]. As observed by atomic force microscopy (AFM) and transmission electron microscopy (TEM), $\mathrm{A}_{6} \mathrm{~K}$, a cationic surfactant-like peptide, could form nanofibers, nanorods, and nanospheres with various length [21]. These nanostructures are quite different with the nanotubes and nanovesicles described in an earlier report. For example, the diameter of these nanostructures is about $10 \mathrm{~nm}$, much smaller than 30 $50 \mathrm{~nm}$; the nanofibers are separated from each other, rather than forming a network by three-way connection. All these differences indicated that these nanostructures are generated by different self-assembling model. The molecular model has also been proposed to explain this alternative self-assembling behavior. As shown in Figure 2(b), peptide molecules pack their hydrophobic tail in a core and expose their hydrophilic head outside, undergoing the formation of cylindrical or spherical micelles to form nanofibers or nanospheres.

The nanostructures formed of surfactant-like peptides could be closely associated with environments. When the $\mathrm{A}_{6} \mathrm{~K}$ solution was spread on mica surface for AFM observation, a kind of membrane-like structure was observed in addition to nanofibers [21]. It is likely that the $\mathrm{A}_{6} \mathrm{~K}$ 

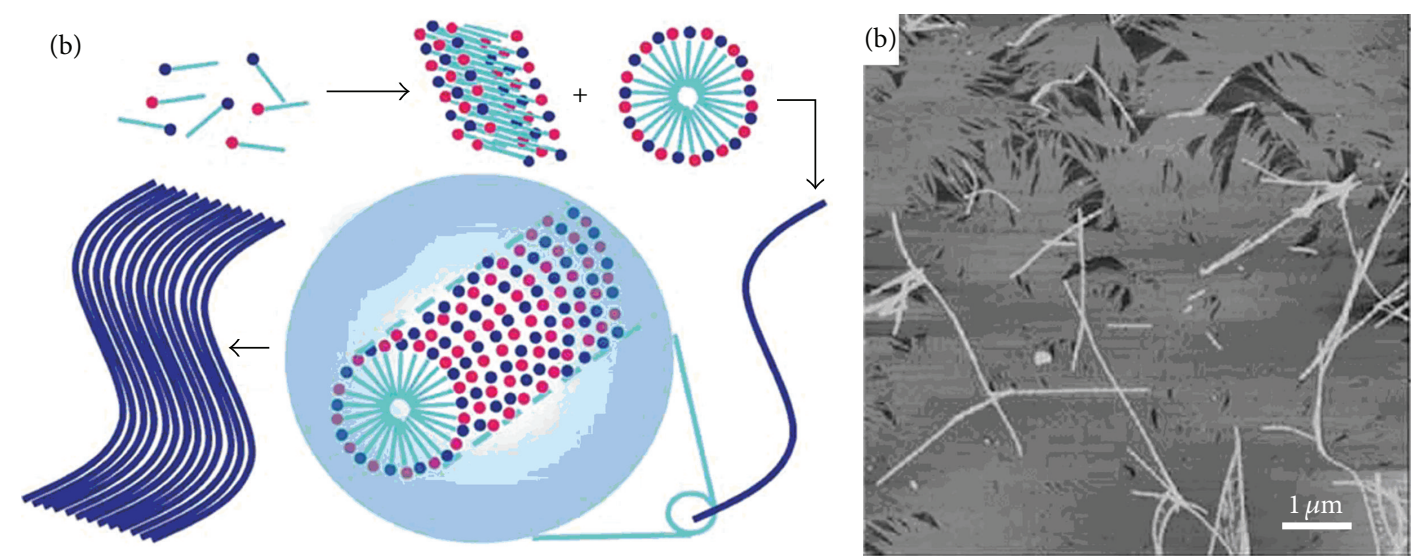

FIGURE 3: Self-assembly of catanionic surfactant-like peptides (reprinted from [25]). (a) Proposed self-assembling model. (b) Well aligned nanoropes observed by AFM.

monomers could attach their positively charged heads to the negatively charged mica surface, while stretching their hydrophobic tails up to air, forming a layer of peptide monomers aligned shoulder by shoulder (Figure 2(c)). In this manner, the peptide monolayer could cover the hydrophilic mica surface and transform it to a hydrophobic one. This interesting self-assembling behavior of $\mathrm{A}_{6} \mathrm{~K}$ on mica surface could act as a very simple technique for surface modification. It is not clear why all kinds of structures fabricated by the same surfactant-like peptide could coexist if no preferential condition is applied, but it is no doubt that the new pathway to form various nanostructures is very helpful to understand the behavior of these self-assembling surfactant-like peptides and to further exploit their potential applications.

\section{Other Surfactant-Like Peptides}

4.1. Catanionic and Zwitterionic Surfactant-Like Peptides. Typical surfactant-like peptides, despite anionic or cationic surfactant-like peptides, often form a mixture of various nanostructures such as nanotubes/nanovesicles and nanofibers/nanospheres. These nanostructures are dynamic and unstable, which becomes an obstacle for their application. For this reason, chemical complementarity should be considered to exploit new surfactant-like peptides with stable selfassembling behavior, which means the preferred formation of a certain type of nanostructure or forming nanostructures with better mechanical/thermal stability at lower critical micelle concentration (CMC). In fact, ionic bond has been proved to be a very important noncovalent force to drive the self-assembling process and determine the properties of self-assembled structures. In order to exploit self-assembling surfactant-like peptides with better stabilities, researchers have made several attempts to introduce ionic bonds into the system of typical surfactant-like peptides.

Catanionic and zwitterionic surfactants are different from typical anionic and cationic surfactants. Catanionic surfactants are the mixed systems of traditional anionic and cationic surfactants [22], and zwitterionic surfactants are the surfactants bearing both positive and negative charges in a single hydrophilic head $[23,24]$. Possibly because of the ionic bonds between oppositely charged heads, these two special groups of surfactants could form structures with better stability compared with typical surfactants [25-28]. Recently, Khoe and colleagues have studied the self-assembling behaviors of several catanionic surfactant-like peptides systems, which were the mixtures of cationic surfactant-like peptide $\mathrm{A}_{6} \mathrm{~K}$ and anionic surfactant-like peptide $\mathrm{A}_{6} \mathrm{D}$ at various ratios [25]. They found that, when $\mathrm{A}_{6} \mathrm{D}$ and $\mathrm{A}_{6} \mathrm{~K}$ were mixed at the ratio of $2: 1$, the catanionic system could form wellordered nanofibers (Figure 3), indicating that in a catanionic system, the interaction between positively and negatively charged heads has significant effects on the self-assembling behavior, pointing out a promising approach to design novel nanomaterials based on mixed surfactant-like peptides.

Recently, we have designed a zwitterionic surfactantlike peptide by simply removing the $\mathrm{C}$-terminal protective amide of $\mathrm{A}_{6} \mathrm{~K}$ and exposing the dissociable carboxyl group [21]. This new peptide, named $\mathrm{A}_{6} \mathrm{~K}^{ \pm}$, could simultaneously bear a positive and a negative charge at its $\mathrm{C}$-terminal. This study showed that $\mathrm{A}_{6} \mathrm{~K}^{ \pm}$could form much longer nanofibers compared with typical anionic or cationic surfactant-like peptides (Figure 4), synergistically driven by the ionic bonds among hydrophilic heads and the hydrophobic interaction among tails. Moreover, the self-assembling structures formed by $\mathrm{A}_{6} \mathrm{~K}^{ \pm}$were also more mechanically and thermally stable than $\mathrm{A}_{6} \mathrm{~K} . \mathrm{A}_{6} \mathrm{~K}^{ \pm}$could also more sensitively respond to the change of environmental $\mathrm{pH}$ and undergo complicated transformation. These results indicated that novel stable and smart nanomaterials could be obtained by designing zwitterionic surfactant-like peptides.

4.2. Bolaamphiphilic Peptides. Different from typical surfactants with only one hydrophilic head, bolaamphiphile, named after a special weapon "bola," has two hydrophilic heads connected by a hydrophobic section [29, 30]. Bolaamphiphiles have been proven to be a category of emerging nanomaterials for their ability to self-assemble into various valuable nanostructures, including membrane-mimetic films for bioactive functions [31, 32], nanotubes for metallic nanowire [33-35], 

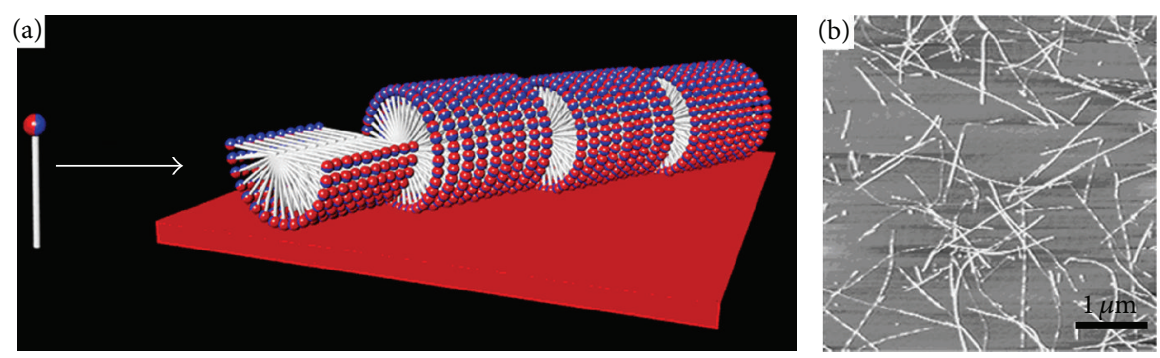

FIGURE 4: Self-assembly of zwitterionic surfactant-like peptides. (a) Proposed self-assembling model. (b) Long nanofibers observed by AFM.

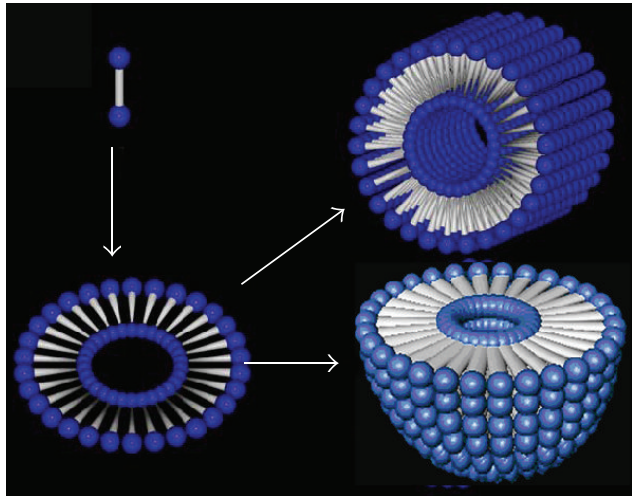

(a)

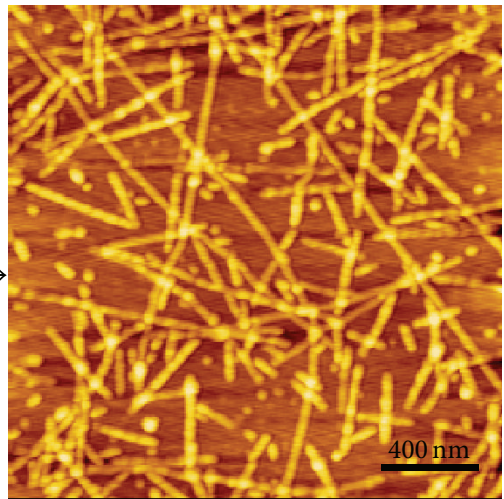

(b)

FIgURE 5: (a) Self-assembling model of bolaamphiphilic peptides. (b) AFM image of bolaamphiphilic peptide $\mathrm{KA}_{6} \mathrm{~K}$.

nanofibers [35], helical ribbons [36], and nanovesicles for drug and gene delivery [37].

By mimicking the structure of these bolaamphiphiles, our laboratory has designed a serial of bolaamphiphilic peptides, which are composed of Gly, Ala, and Val as their hydrophobic sections and charged Lys or Asp as their hydrophilic heads [38]. By adjusting hydrophobic or hydrophilic amino acids, the length and hydrophobicity of the bolaamphiphilic peptide molecule could be controlled to generate different self-assembling nanostructures, such as nanofibers or nanospheres with hydrophilic core and surface (Figure 5). Different from the nanotubes and nanovesicles with diameter about $30 \sim 50 \mathrm{~nm}$ formed by typical surfactant-like peptides, these nanostructures formed by bolaamphiphilic peptides have a diameter less than $10 \mathrm{~nm}$, The smaller size and the hydrophilic core might be important features for their application as cell-targeting carriers for hydrophilic molecules such as DNA, RNA, and some hydrophilic drugs. Furthermore, it has been found that after destroyed by sonication, the nanostructures formed by bolaamphiphilic peptides could undergo a slow process of reassembly to recovery, which is also an important feature for the potential of encapsulating small molecular components. Recently, bolaamphiphilic peptides with alkyl group as their hydrophobic sections have also been designed, which could self-assemble into nanofibers with hydrophilic core and surface [39].

4.3. Surfactant-Like Peptides with Ameliorated Geometrical Shape. It is well known that chemical complementarity and geometrical compatibility are two basic elements for molecular self-assembly [40, 41]. For this reason, when ameliorating the structure of self-assembling molecules for better properties, geometrical compatibility should be considered. Our laboratory has designed several surfactantlike peptides with different geometrical shapes in order to study the effect of geometrical shape on the self-assembling behavior of surfactant-like peptides [42]. Briefly, by changing the amino acids, which have different spatial structures, $\mathrm{AVK}^{ \pm}$(Ac-AAAVVVK) has a wedge-like shape, and $\mathrm{AGK}^{ \pm}$ (Ac-AAAGGGK) has a shape of inverted wedge. Compared with $\mathrm{A}_{6} \mathrm{~K}^{ \pm}$(Ac-AAAAAAK), which has a straight shape, the wedge-shaped $\mathrm{AVK}^{ \pm}$undergoes a more homogeneous selfassembling behavior to form much longer nanofibers, and these nanofibers are relatively more stable and less prone to be transformed to nanospheres, while the self-assembling behavior of $\mathrm{A}_{6} \mathrm{~K}^{ \pm}$is very dynamic and it tends to form mixture of nanofibers with various lengths and nanospheres. On the contrary, $\mathrm{AGK}^{ \pm}$, with inverted-wedge-like shape, couldn't undergo self-assembly in aqueous solution but could selfassemble into nanofibers with various lengths in nonpolar solvent.

As shown in Figure 6, 3D molecular models have been proposed to illuminate the effects of geometrical shape on self-assembling behaviors. For $\mathrm{AVK}^{ \pm}$, the wedge-like shape could efficiently reduce the spatial encumbrance when $\mathrm{AVK}^{ \pm}$molecules self-assemble into micelles, so that they can form stable cylindrical micelle nanofibers. For $\mathrm{AGK}^{ \pm}$, the inverted-wedge-like shape promotes them to embed the small 


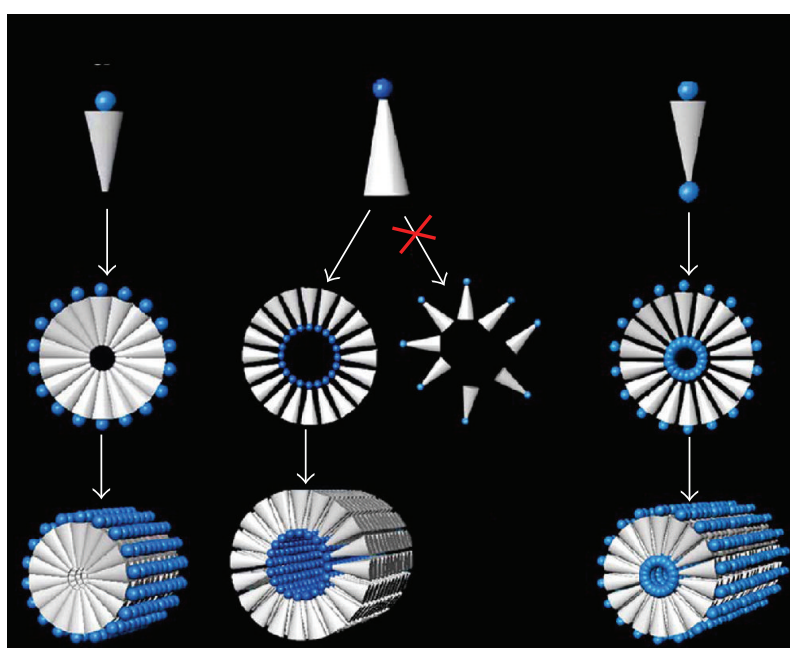

(a)

(b)

(c)

FIGURE 6: Self-assembly of surfactant-like peptides with different geometrical shape. (a) Wedge-shaped surfactant-like peptides form cylindrical micelle nanofibers in water solution. (b) Reversed-wedge-shaped surfactant-like peptide forms reverse micelle nanofibers in nonpolar environment. (c) Wedge-shaped bolaamphiphilic peptides form cylindrical micelle nanofibers in water solution.

hydrophilic head inside and expose the large hydrophobic tail to the nonpolar solution, finally forming reversed micelle nanofibers.

When the geometrical shape of the hydrophobic tail of a peptide was concerned, the property of the hydrophilic head seems to be less important. Similar to the self-assembling behavior of $\mathrm{AVK}^{ \pm}$, it has also been found that a negatively charged surfactant-like peptide $\mathrm{A}_{3} \mathrm{~V}_{3} \mathrm{D}$ with wedge-like shape could also form smooth long nanofibers [43]. On the other hand, more hydrophobic amino acids could also be used to design surfactant-like peptide with geometrical shape effect. For example, a cone-shaped amphiphilic peptide AcGAVILRR- $\mathrm{NH}_{2}$ could self-assemble into nanodonut structure through the fusion or elongation of spherical micelles [44]. These studies suggested that geometrical shape plays a crucial role in controlling the self-assembling behavior of surfactant-like peptides to form certain nanostructure.

As for the typical surfactant-like peptide system described above, geometrical shape could also greatly affect the selfassembling structure of bolaamphiphilic peptide. Our group has designed a novel bolaamphiphilic KGGAAVVK, which exhibited a wedge-like shape for the increasing size from Gly to Val. Unlike $\mathrm{KA}_{6} \mathrm{~K}$ with straight shape which self-assemble into mixture of nanofibers and nanospheres, KGGAAVVK could selectively form long and smooth nanofibers [43]. Similar to the model proposed for typical surfactant-like peptide, the wedge-like shape of the peptide was also regarded as an important factor for the formation of such long nanofibers.

Besides those surfactant-like peptides totally composed of natural amino acids introduced above, some other similar self-assembling peptides containing special groups have also been studied in recent years. One of the most extensively studied categories is peptide amphiphiles developed by Webber et al. $[45,46]$. In these peptide amphiphiles, a typical molecule contains an alkyl chain as its hydrophobic tail and a functional peptide group as its hydrophilic head. By forming micellar nanofibers and exposing functional peptide groups outside, peptide amphiphiles have been proved to be potential nanomaterials which have been widely used in many fields including 3D cell culture and tissue engineering. Moreover, surfactant-like peptide has also been combined with traditional surfactant and shown novel self-assembling behavior which could be used as template for Au nanoparticles [47].

\section{Applications of Surfactant-Like Peptides}

5.1. Surfactant-Like Peptides Stabilizing Membrane Protein. According to the computational analyses of completely sequenced genomes, about one-third of all cellular proteins are membrane proteins, which contain at least one transmembrane domain and have critical roles in many important life activities such as cell signaling, cell migration and movement, energy transformation, and substance transport [48]. However, most molecular structures of membrane proteins remain elusive, which is in sharp contrast to their great important functions in cells. The reason for this paradox is that natural membrane proteins are usually embedded in lipid bilayer, traditional purification, and crystallization methods for water-soluble proteins will remove lipids from membrane proteins, and affect the solubility and conformation stability of membrane proteins. In the past decades, many traditional surfactants such as detergents and lipids have been used for the stabilization, purification, and crystallization of membrane proteins; however, due to the complexity of membrane protein-detergent-lipid interactions, the efficacy is still far from satisfaction. Thus, the discovery and design of novel surfactants are acutely necessary to facilitate membrane protein purification and crystallization for structural studies.

Recently, surfactant-like peptides have shown promising potential in the study of membrane proteins. These surfactant-like peptides have structural properties similar to lipid: peptide molecule has a hydrophobic tail composed of several consecutive hydrophobic amino acids and a hydrophilic head composed of one or two hydrophilic amino acids. Based on the molecular structure, surfactantlike peptides could bind to the hydrophobic section of a membrane protein by using their hydrophobic tails and sequester it from water, preventing membrane protein from denaturation.

G protein-coupled receptors (GPCRs) are a large class of membrane proteins, which play a crucial role in cell signaling pathways and become a worldwide hotspot in the pharmaceutical industries. Unfortunately, the structures of most GPCRs remain elusive. To study the structure and function of diverse GPCRs, researchers have stabilized these membrane proteins with surfactant-like peptides. For example, Zhao has chosen bovine rhodopsin, one of GPCRs, and investigated the stabilization of bovine rhodopsin in solution by using a new class of surfactant-like peptides [49]. As shown in Figure 7, these surfactant-like peptides not only enhance the stability of bovine rhodopsin in the presence of lipids and the 


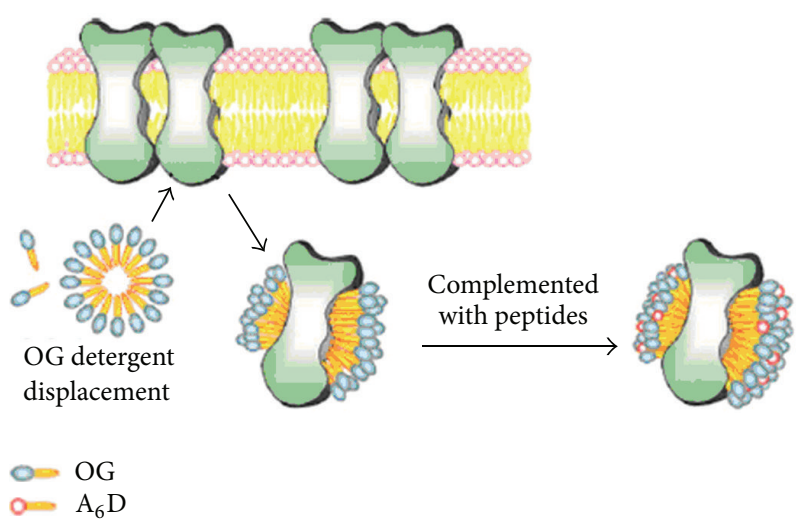

(a)

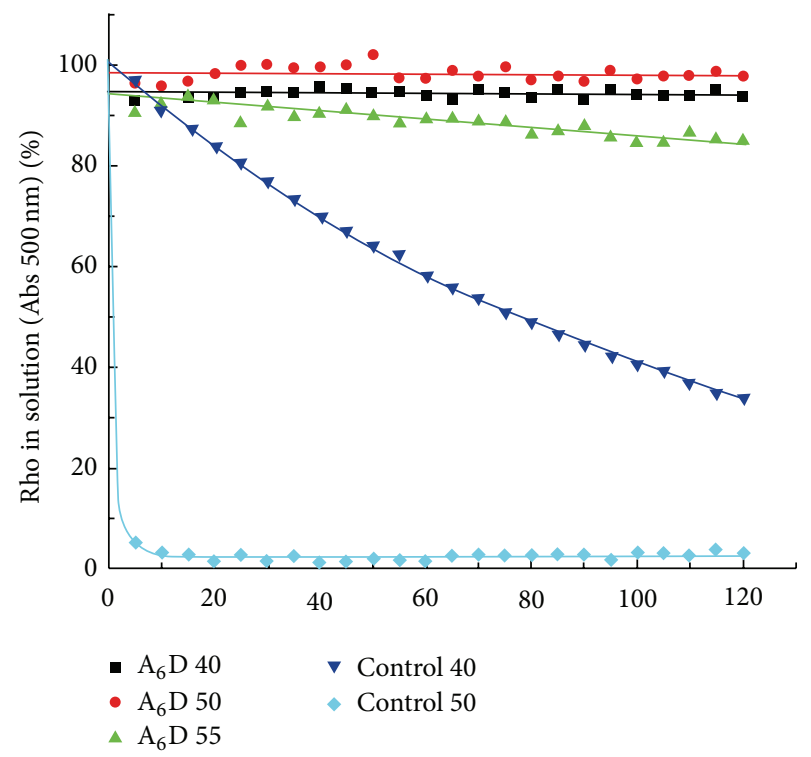

(b)

FIGURE 7: Surfactant-like peptides stabilize membrane proteins. (a) The proposed mechanism of surfactant-like peptide $\mathrm{A}_{6} \mathrm{D}$ stabilizing membrane proteins (reprinted from [49]). (b) Stability of rhodopsin (Rho) in the absence of OG at different temperatures. Half-life of rhodopsin was as follows: not available in $2.5 \mathrm{mM} \mathrm{A}_{6} \mathrm{D}$ at $40^{\circ} \mathrm{C}, 50^{\circ} \mathrm{C}$, and $55^{\circ} \mathrm{C} ; 101 \mathrm{~min}$ in control solution $\left(1.25 \mathrm{mM} \mathrm{A}_{6} \mathrm{D} / 1 \% \mathrm{OG}\right)$ at $40^{\circ} \mathrm{C}$; $<5 \mathrm{~min}$ in control solution at $50^{\circ} \mathrm{C}$ (reprinted from [49]).

common surfactants $\mathrm{n}$-dodecyl- $\beta$-D-maltoside and octyl$\mathrm{D}$-glucoside but also effectively stabilize rhodopsin under thermal denaturation conditions even after the lipids were removed.

Additionally, researchers have investigated whether photosynthetic complexes could be effectively stabilized by surfactant-like peptides. Photosynthetic complexes are crucial proteins located in the membrane of chloroplast and are capable of transforming solar energy with amazing high efficacy, which promotes photosynthetic complexes to be made into nanodevices of solar biobattery as a safe and sustainable energy resource. To develop such solar biobattery, the stabilizing of photosynthetic complexes with bioactivity is the first step. Researchers have certified the feasibility of stabilizing photosynthetic complexes with designed surfactantlike peptides. The fluorescence spectrum of photosystem I (PSI) stabilized by surfactant-like peptides had no significant difference compared with PSI directly extracted from leaves of spinach. Surprisingly, PSI could even maintain its bioactivity on a dry surface in a considerably long period by the protection of an anionic surfactant-like peptide $\mathrm{V}_{6} \mathrm{D}$ [48].

\subsection{Surfactant-Like Peptides as Drug and Gene Carriers.} Since the surfactant-like peptide molecules can be easily designed and modified to form various nanostructures, they can be easily tailored for drug or gene delivery. These peptides have a unique amphiphilic structure, and the hydrophobic tail could promote surfactant-like peptides to self-assemble into the nanostructure with a hydrophobic core, which has potential to encapsulate water-insoluble molecules and deliver drugs and other biological molecules. On the other hand, the hydrophilic head could be modified as functional group for cell-targeting. Recently, a surfactant-like peptide with fatty tail was investigated as a hydrophobic drug carrier [50].

So far, gene therapy endeavors have still lacked optimal DNA delivery systems that are highly efficient, nontoxic, and simple to produce in large scales. Recently, it was found that a number of cationic surfactant-like peptides have great potential for gene delivery [51]. Compared with hydrophobic drug delivery, negatively charged DNA is expected to bind to the positively charged head of surfactant-like peptides. For example, cholesterol-conjugated HR15 and HR20 oligopeptides were synthesized, which were able to self-assemble into cationic micelles. The formation of the micelles increased local density of cationic charge, leading to greater DNA binding efficiency and thus higher gene transfection efficiency in both HepG2 and HEK293 cell lines [51]. Furthermore, surfactant-like peptide FA32 could form small micelles with particle size of about $100 \mathrm{~nm}$, which could deliver hydrophobic drug DOX inside the cells efficiently. Meanwhile, these micelles could deliver DNA into HepG2 cells with high efficiency. These findings suggest that surfactant-like peptides could have the great potential to deliver hydrophobic drug and gene simultaneously into the same cells to achieve synergistic therapeutic effect [52].

5.3. Surfactant-Like Peptides for Tissue Engineering. Recently, tissue engineering, aimed at resolving the problems in organ repairing and tissue regeneration, has become an important strategy in modern biomedical technology. Generally, tissue engineering requires two complementary ingredients: suitable cells and compatible scaffolds [53]. It would be necessary to apply compatible biological scaffolds, which could stimulate and promote cell differentiation, as well as regenerating tissues without harm. For this purpose, many scaffoldforming self-assembling peptides have been reported [54$60]$. However, the use of scaffold materials is inevitably accompanied by some side effects such as fibrosis and inflammatory response. Alternatively, another kind of scaffold-free tissue engineering strategy, cell sheet technology, has been 
(a)
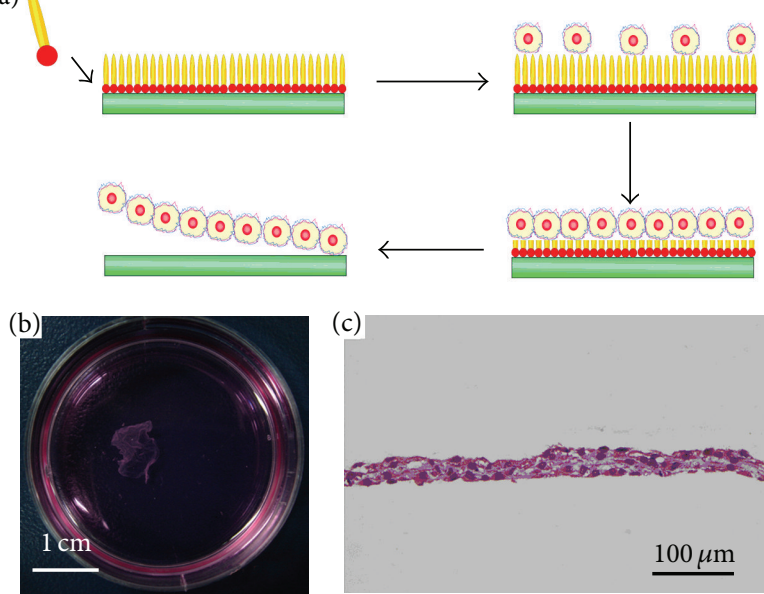

FIGURE 8: Cell sheet technology based on surfactant-like peptides. (a) Mechanism of harvesting cell sheet on mica surface modified by surfactant-like peptides. (b) Harvested cell sheet. (c) HE staining of cell sheet (reprinted from [65]).

widely investigated in recent years. This is a novel technique in which a sheet-like integration of cells is cultured and harvested by certain methods. Cell sheet could maintain cellto-cell connections and the cellular matrix, and the bionic structures could promote cell sheet to regenerate organs as a small piece of artificial tissue [61-64].

In recent years, this scaffold-free tissue engineering strategy has received rapid development and become an important complementary to traditional scaffold-involved strategy. In this field, surfactant-like peptides as a novel type of surface modifying molecules have shown its potential. It was found that $A_{6} K$, a cationic surfactant-like peptide, could self-assemble on mica surface to form a monolayer. In this way, a hydrophilic mica surface could be modified into a hydrophobic one, which is suitable for cell adhesion and growth. Along with the growth of cultured cells, $\mathrm{A}_{6} \mathrm{~K}$ peptide composed of natural L-amino acids could be gradually biodegraded, and the hydrophilic mica surface could be reexposed, which is adverse for cell adhesion, and thus an integrate of cell sheet could be easily released from the mica surface (Figure 8 ) [65]. This novel cell sheet technique based on surfactant-like peptide is very simple, effective, and safe and may become a very important technique for the development of cell sheet technique.

5.4. Surfactant-Like Peptides as Template for Nanofabrication. For the development of nanoscience and nanotechnology, fabricating nanostructures and nanodevices is the first important task. In this field, many self-assembling structures have been successfully used as template for nanofabrication. Recently, a surfactant-like peptide AGD, which could undergo self-assembly in nonpolar solvent system, has shown its potential for such application. This peptide was designed to have a shape like an inverted wedge which prevented it to self-assemble in aqueous solution. But in nonpolar mixture of water and tetrahydrofuran, and with the existence of copper ion, the peptide could self-assemble into nanorings by forming reversed micelle [66]. It was proved that copper ions were bound with the negatively charged head of the peptide and embedded in the core of the nanoring. In this manner, the peptide could be applied as a template for fabricating novel metallic nanostructures.

\section{Conclusion and Outlook}

The field of the design and application of surfactant-like peptides has been developed very rapidly in recent years. These peptides are easy to be designed and synthesized, which could guarantee their quality and purity; moreover, the biocompatibility of surfactant-like peptides makes them perfect materials for biological and biomedical applications. Their applications in the fields of membrane protein stabilizing and tissue engineering have proved their great potential. On the other hand, the chemical structure and self-assembling mechanism of surfactant-like peptides are very simple and clear; thus, surfactant-like peptides also provide a simple selfassembling model to study the folding and assembling of natural proteins, which might be very helpful to understand the mechanism of protein conformational diseases.

However, challenge remains when considering the practical application of the family of surfactant-like peptide. One reason might be due to the complicated self-assembling behavior of surfactant-like peptides which were highly sensitive to environmental parameters such as $\mathrm{pH}$, ion strength, and peptide concentration. Although several attempts have been tried to obtain controllable self-assembling nanostructures, further investigations are still needed to be carried out to clarify the molecular mechanism for the self-assembling of surfactant like peptides, as well as their interaction with other biological molecules. This may be of great importance to exploit the application of surfactant-like peptides, especially as potential drug delivery nanomaterials.

\section{Acknowledgments}

This work was supported in part by the National $985 \mathrm{Key}$ Project of Sichuan University of the Education Ministry of China. The authors thank Professor Shuguang Zhang (Massachusetts Institute of Technology) for his permission to use his figures in this paper.

\section{References}

[1] S. Winkler, S. Szela, P. Avtges, R. Valluzzi, D. A. Kirschner, and D. Kaplan, "Designing recombinant spider silk proteins to control assembly," International Journal of Biological Macromolecules, vol. 24, no. 2-3, pp. 265-270, 1999.

[2] S. Zhang, T. C. Holmes, C. M. DiPersio, R. O. Hynes, X. Su, and A. Rich, "Self-complementary oligopeptide matrices support mammalian cell attachment," Biomaterials, vol. 16, no. 18, pp. 1385-1393, 1995.

[3] T. C. Holmes, S. de Lacalle, X. Su, G. Liu, A. Rich, and S. Zhang, "Extensive neurite outgrowth and active synapse formation on self-assembling peptide scaffolds," Proceedings of the National 
Academy of Sciences of the United States of America, vol. 97, no. 12 , pp. 6728-6733, 2000.

[4] J. Kisiday, M. Jin, B. Kurz et al., "Self-assembling peptide hydrogel fosters chondrocyte extracellular matrix production and cell division: implications for cartilage tissue repair," Proceedings of the National Academy of Sciences of the United States of America, vol. 99, no. 15, pp. 9996-10001, 2002.

[5] M. A. Bokhari, G. Akay, S. Zhang, and M. A. Birch, "The enhancement of osteoblast growth and differentiation in vitro on a peptide hydrogel-PolyHIPE polymer hybrid material," Biomaterials, vol. 26, no. 25, pp. 5198-5208, 2005.

[6] R. G. Ellis-Behnke, Y.-X. Liang, S.-W. You et al., "Nano neuro knitting: peptide nanofiber scaffold for brain repair and axon regeneration with functional return of vision," Proceedings of the National Academy of Sciences of the United States of America, vol. 103, no. 13, pp. 5054-5059, 2006.

[7] S. Zhang, F. Gelain, and X. Zhao, "Designer self-assembling peptide nanofiber scaffolds for 3D tissue cell cultures," Seminars in Cancer Biology, vol. 15, no. 5, pp. 413-420, 2005.

[8] S. Koutsopoulos and S. Zhang, "Long-term three-dimensional neural tissue cultures in functionalized self-assembling peptide hydrogels, Matrigel and Collagen I," Acta Biomaterialia, vol. 9, no. 2, pp. 5162-5169, 2013.

[9] C. E. Semino, J. Kasahara, Y. Hayashi, and S. Zhang, "Entrapment of migrating hippocampal neural cells in three-dimensional peptide nanofiber scaffold," Tissue Engineering, vol. 10, no. 3-4, pp. 643-655, 2004.

[10] M. Nune, P. Kumaraswamy, U. M. Krishnan, and S. Sethuraman, "Self-assembling peptide nanofibrous scaffolds for tissue engineering: novel approaches and strategies for effective functional regeneration," Current Protein \& Peptide Science, vol. 14, no. 1, pp. 70-84, 2013.

[11] T. Y. Cheng, M. H. Chen, W. H. Chang, M. Y. Huang, and T. W. Wang, "Neural stem cells encapsulated in a functionalized self-assembling peptide hydrogel for brain tissue engineering," Biomaterials, vol. 34, no. 8, pp. 2005-2016, 2013.

[12] R. Bawa, S.-Y. Fung, A. Shiozaki et al., "Self-assembling peptidebased nanoparticles enhance cellular delivery of the hydrophobic anticancer drug ellipticine through caveolae-dependent endocytosis," Nanomedicine, vol. 8, no. 5, pp. 647-654, 2012.

[13] S. Koutsopoulos and S. Zhang, "Two-layered injectable selfassembling peptide scaffold hydrogels for long-term sustained release of human antibodies," Journal of Controlled Release, vol. 160, no. 3, pp. 451-458, 2012.

[14] S. Deshayes, K. Konate, A. Rydstrom et al., "Self-assembling peptide-based nanoparticles for siRNA delivery in primary cell lines," Small, vol. 8, no. 14, pp. 2184-2188, 2012.

[15] X. Zhao, "Design of self-assembling surfactant-like peptides and their applications," Current Opinion in Colloid and Interface Science, vol. 14, no. 5, pp. 340-348, 2009.

[16] S. Vauthey, S. Santoso, H. Gong, N. Watson, and S. Zhang, "Molecular self-assembly of surfactant-like peptides to form nanotubes and nanovesicles," Proceedings of the National Academy of Sciences of the United States of America, vol. 99, no. 8, pp. 5355-5360, 2002.

[17] S. Santoso, W. Hwang, H. Hartman, and S. Zhang, "Self-assembly of surfactant-like peptides with variable glycine tails to form nanotubes and nanovesicles," Nano Letters, vol. 2, no. 7, pp. 687-691, 2002.

[18] G. Maltzahn, S. Vauthey, S. Santoso, and S. Zhang, "Positively charged surfactant-like peptides self-assemble into nanostructures," Langmuir, vol. 19, no. 10, pp. 4332-4337, 2003.
[19] S. J. Yang and S. Zhang, "Self-assembling behavior of designer lipid-like peptides," Supramolecular Chemistry, vol. 18, no. 5, pp. 389-396, 2006.

[20] A. Nagai, Y. Nagai, H. Qu, and S. Zhang, "Dynamic behaviors of lipid-like self-assembling peptide $\mathrm{A}_{6} \mathrm{D}$ and $\mathrm{A}_{6} \mathrm{~K}$ nanotubes," Journal of Nanoscience and Nanotechnology, vol. 7, no. 7, pp. 2246-2252, 2007.

[21] F. Qiu, Y. Chen, and X. Zhao, "Comparative studies on the selfassembling behaviors of cationic and catanionic surfactant-like peptides," Journal of Colloid and Interface Science, vol. 336, no. 2, pp. 477-484, 2009.

[22] P. Jokela, B. Jönsson, and A. Khan, "Phase equilibria of catanionic surfactant-water systems," Journal of Physical Chemistry, vol. 91, no. 12, pp. 3291-3298, 1987.

[23] K. Tsubone and N. Uchida, "Syntheses of 2-(N-alkyl-N,Ndimethylammonio)ethyl hydrogen phosphates and their physicochemical properties," Journal of the American Oil Chemists' Society, vol. 67, no. 3, pp. 149-153, 1990.

[24] L. M. Camillo, B. Sesta, M. G. Bonicelli, and G. F. Ceccaroni, "Phase diagram of the binary system water-(dodecyldimethylammonio)propanesulfonate," Langmuir, vol. 6, no. 4, pp. 728-731, 1990.

[25] U. Khoe, Y. Yang, and S. Zhang, "Synergistic effect and hierarchical nanostructure formation in mixing two designer lipid-like peptide surfactants Ac- $\mathrm{A}_{6} \mathrm{D}-\mathrm{OH}$ and $\mathrm{Ac}-\mathrm{A}_{6} \mathrm{~K}-\mathrm{NH}_{2}$," Macromolecular Bioscience, vol. 8, no. 11, pp. 1060-1067, 2008.

[26] B. F. B. Silva and E. F. Marques, "Thermotropic behavior of asymmetric chain length catanionic surfactants: the influence of the polar head group," Journal of Colloid and Interface Science, vol. 290, no. 1, pp. 267-274, 2005.

[27] J. Wang, A. Song, X. Jia, J. Hao, W. Liu, and H. Hoffmann, "Two routes to vesicle formation: metal-ligand complexation and ionic interactions," Journal of Physical Chemistry B, vol. 109, no. 22, pp. 11126-11134, 2005.

[28] B. Yue, C.-Y. Huang, M.-P. Nieh, C. J. Glinka, and J. Katsaras, "Highly stable phospholipid unilamellar vesicles from spontaneous vesiculation: a DLS and SANS study," Journal of Physical Chemistry B, vol. 109, no. 1, pp. 609-616, 2005.

[29] J.-H. Fuhrhop and J. Mathieu, "Routes to functional vesicle membranes without proteins," Angewandte Chemie, vol. 23, no. 2, pp. 100-113, 1984.

[30] J.-H. Fuhrhop, H.-H. David, J. Mathieu, U. Liman, H.-J. Winter, and E. Boekema, "Bolaamphiphiles and monolayer lipid membranes made from 1,6,19,24-tetraoxa-3,21-cyclohexatriacontadiene-2,5,20,23-tetrone," Journal of the American Chemical Society, vol. 108, no. 8, pp. 1785-1791, 1986.

[31] X.-L. Sun, N. Biswas, T. Kai, Z. Dai, R. A. Dluhy, and E. L. Chaikof, "Membrane-mimetic films of asymmetric phosphatidylcholine lipid bolaamphiphiles," Langmuir, vol. 22, no. 3, pp. 1201-1208, 2006.

[32] L. M. Cameron, T. M. Fyles, and C.-W. Hu, "Synthesis and membrane activity of a bis(metacyclophane)bolaamphiphile," Journal of Organic Chemistry, vol. 67, no. 5, pp. 1548-1553, 2002.

[33] C. Zhan, P. Gao, and M. Liu, "Self-assembled helical sphericalnanotubes from an L-glutamic acid based bolaamphiphilic low molecular mass organogelator," Chemical Communications, no. 4, pp. 462-464, 2005.

[34] P. Gao and M. Liu, "Compression induced helical nanotubes in a spreading film of a bolaamphiphile at the air/water interface," Langmuir, vol. 22, no. 16, pp. 6727-6729, 2006. 
[35] P. Gao, C. Zhan, and M. Liu, "Controlled synthesis of doubleand multiwall silver nanotubes with template organogel from a bolaamphiphile," Langmuir, vol. 22, no. 2, pp. 775-779, 2006.

[36] J. Song, Q. Cheng, and R. C. Stevens, "Morphological manipulation of bolaamphiphilic polydiacetylene assemblies by controlled lipid doping," Chemistry and Physics of Lipids, vol. 114, no. 2, pp. 203-214, 2002.

[37] V. Weissig and V. P. Torchilin, "Mitochondriotropic cationic vesicles: a strategy towards mitochondrial gene therapy," Current Pharmaceutical Biotechnology, vol. 1, no. 4, pp. 325-346, 2000.

[38] F. Qiu, Y. Chen, C. Tang et al., "De novo design of a bolaamphiphilic peptide with only natural amino acids," Macromolecular Bioscience, vol. 8, no. 11, pp. 1053-1059, 2008.

[39] R. C. Claussen, B. M. Rabatic, and S. I. Stupp, "Aqueous self-assembly of unsymmetric peptide bolaamphiphiles into nanofibers with hydrophilic cores and surfaces," Journal of the American Chemical Society, vol. 125, no. 42, pp. 12680-12681, 2003.

[40] S. Zhang, "Emerging biological materials through molecular self-assembly," Biotechnology Advances, vol. 20, no. 5-6, pp. 321339, 2002.

[41] X. Zhao and S. Zhang, "Designer self-assembling peptide materials," Macromolecular Bioscience, vol. 7, no. 1, pp. 13-22, 2007.

[42] Y. Chen, F. Qiu, Y. Lu, Y.-K. Shi, and X. Zhao, "Geometrical shape of hydrophobic section determines the self-assembling structure of peptide detergents and bolaamphiphilic peptides," Current Nanoscience, vol. 5, no. 1, pp. 69-74, 2009.

[43] Y.-Z. Chen, F. Qiu, and X.-J. Zhao, "Self-assembling structure and mechanism of a wedge-shaped peptide detergent $A_{3} V_{3} D$," Gaodeng Xuexiao Huaxue Xuebao/Chemical Journal of Chinese Universities, vol. 30, no. 7, pp. 1337-1341, 2009.

[44] U. Khoe, Y. Yang, and S. Zhang, "Self-assembly of nanodonut structure from a cone-shaped designer lipid-like peptide surfactant," Langmuir, vol. 25, no. 7, pp. 4111-4114, 2009.

[45] M. J. Webber, J. Tongers, M.-A. Renault, J. G. Roncalli, D. W. Losordo, and S. I. Stupp, "Development of bioactive peptide amphiphiles for therapeutic cell delivery," Acta Biomaterialia, vol. 6 , no. 1, pp. 3-11, 2010.

[46] M. J. Webber, J. A. Kessler, and S. I. Stupp, "Emerging peptide nanomedicine to regenerate tissues and organs," Journal of Internal Medicine, vol. 267, no. 1, pp. 71-88, 2010.

[47] Y. Dou, H. Xu, and J. Hao, "Self-assembly and accurate preparation of Au nanoparticles in the aqueous solution of a peptide $\mathrm{A}_{6} \mathrm{D}$ and a zwitterionic $\mathrm{Cl}_{4} \mathrm{DMAO}$, Soft Matter, vol. 9, no. 23, pp. 5572-5580, 2013.

[48] P. Kiley, X. Zhao, M. Vaughn, M. A. Baldo, B. D. Bruce, and S. Zhang, "Self-assembling peptide detergents stabilize isolated photosystem I on a dry surface for an extended time," PLoS Biology, vol. 3, no. 7, article e230, 2005.

[49] X. Zhao, Y. Nagai, P. J. Reeves, P. Kiley, H. G. Khorana, and S. Zhang, "Designer short peptide surfactants stabilize G proteincoupled receptor bovine rhodopsin," Proceedings of the National Academy of Sciences of the United States of America, vol. 103, no. 47, pp. 17707-17712, 2006.

[50] J.-X. Chen, H.-Y. Wang, C. Li, K. Han, X.-Z. Zhang, and R.$\mathrm{X}$. Zhuo, "Construction of surfactant-like tetra-tail amphiphilic peptide with RGD ligand for encapsulation of porphyrin for photodynamic therapy," Biomaterials, vol. 32 , no. 6 , pp. $1678-$ 1684, 2011.
[51] X. D. Guo, F. Tandiono, N. Wiradharma et al., "Cationic micelles self-assembled from cholesterol-conjugated oligopeptides as an efficient gene delivery vector," Biomaterials, vol. 29, no. 36, pp. 4838-4846, 2008.

[52] N. Wiradharma, Y. W. Tong, and Y.-Y. Yang, "Self-assembled oligopeptide nanostructures for co-delivery of drug and gene with synergistic therapeutic effect," Biomaterials, vol. 30, no. 17, pp. 3100-3109, 2009.

[53] R. Langer and J. P. Vacanti, “Tissue engineering," Science, vol. 260, no. 5110, pp. 920-926, 1993.

[54] M. J. Webber, J. Tongers, M.-A. Renault, J. G. Roncalli, D. W. Losordo, and S. I. Stupp, "Development of bioactive peptide amphiphiles for therapeutic cell delivery," Acta Biomaterialia, vol. 6, no. 1, pp. 3-11, 2010.

[55] M. J. Webber, J. A. Kessler, and S. I. Stupp, "Emerging peptide nanomedicine to regenerate tissues and organs," Journal of Internal Medicine, vol. 267, no. 1, pp. 71-88, 2010.

[56] S. M. Standley, D. J. Toft, H. Cheng et al., "Induction of cancer cell death by self-assembling nanostructures incorporating a cytotoxic peptide," Cancer Research, vol. 70, no. 8, pp. 30203026, 2010

[57] K. Rajangam, M. S. Arnold, M. A. Rocco, and S. I. Stupp, "Peptide amphiphile nanostructure-heparin interactions and their relationship to bioactivity," Biomaterials, vol. 29, no. 23, pp. 3298-3305, 2008.

[58] J. D. Tovar, B. M. Rabatic, and S. I. Stupp, "Conducting polymers confined within bioactive peptide amphiphile nanostructures," Small, vol. 3, no. 12, pp. 2024-2028, 2007.

[59] E. Beniash, J. D. Hartgerink, H. Storrie, J. C. Stendahl, and S. I. Stupp, "Self-assembling peptide amphiphile nanofiber matrices for cell entrapment," Acta Biomaterialia, vol. 1, no. 4, pp. 387397, 2005.

[60] G. A. Silva, C. Czeisler, K. L. Niece et al., "Selective differentiation of neural progenitor cells by high-epitope density nanofibers," Science, vol. 303, no. 5662, pp. 1352-1355, 2004.

[61] A. Ito, H. Jitsunobu, Y. Kawabe, and M. Kamihira, "Construction of heterotypic cell sheets by magnetic force-based 3-D coculture of HepG2 and NIH3T3 cells," Journal of Bioscience and Bioengineering, vol. 104, no. 5, pp. 371-378, 2007.

[62] K. Ohashi, T. Yokoyama, M. Yamato et al., "Engineering functional two- and three-dimensional liver systems in vivo using hepatic tissue sheets," Nature Medicine, vol. 13, no. 7, pp. 880-885, 2007.

[63] Y. Miyahara, N. Nagaya, M. Kataoka et al., "Monolayered mesenchymal stem cells repair scarred myocardium after myocardial infarction," Nature Medicine, vol. 12, no. 4, pp. 459-465, 2006.

[64] H. Kobayashi, T. Shimizu, M. Yamato et al., "Fibroblast sheets co-cultured with endothelial progenitor cells improve cardiac function of infarcted hearts," Journal of Artificial Organs, vol. 11, no. 3, pp. 141-147, 2008.

[65] F. Qiu, Y. Chen, J. Cheng, C. Wang, H. Xu, and X. Zhao, "A simple method for cell sheet fabrication using mica surfaces grafted with peptide detergent $\mathrm{A}_{6} \mathrm{~K}$," Macromolecular Bioscience, vol.10, no. 8, pp. 881-886, 2010.

[66] F. Qiu, Y. Chen, C. Tang, J. Cheng, and X. Zhao, "Formation of reversed micelle nanoring by a designed surfactant-like peptide," Nano, vol. 7, no. 4, 2012. 

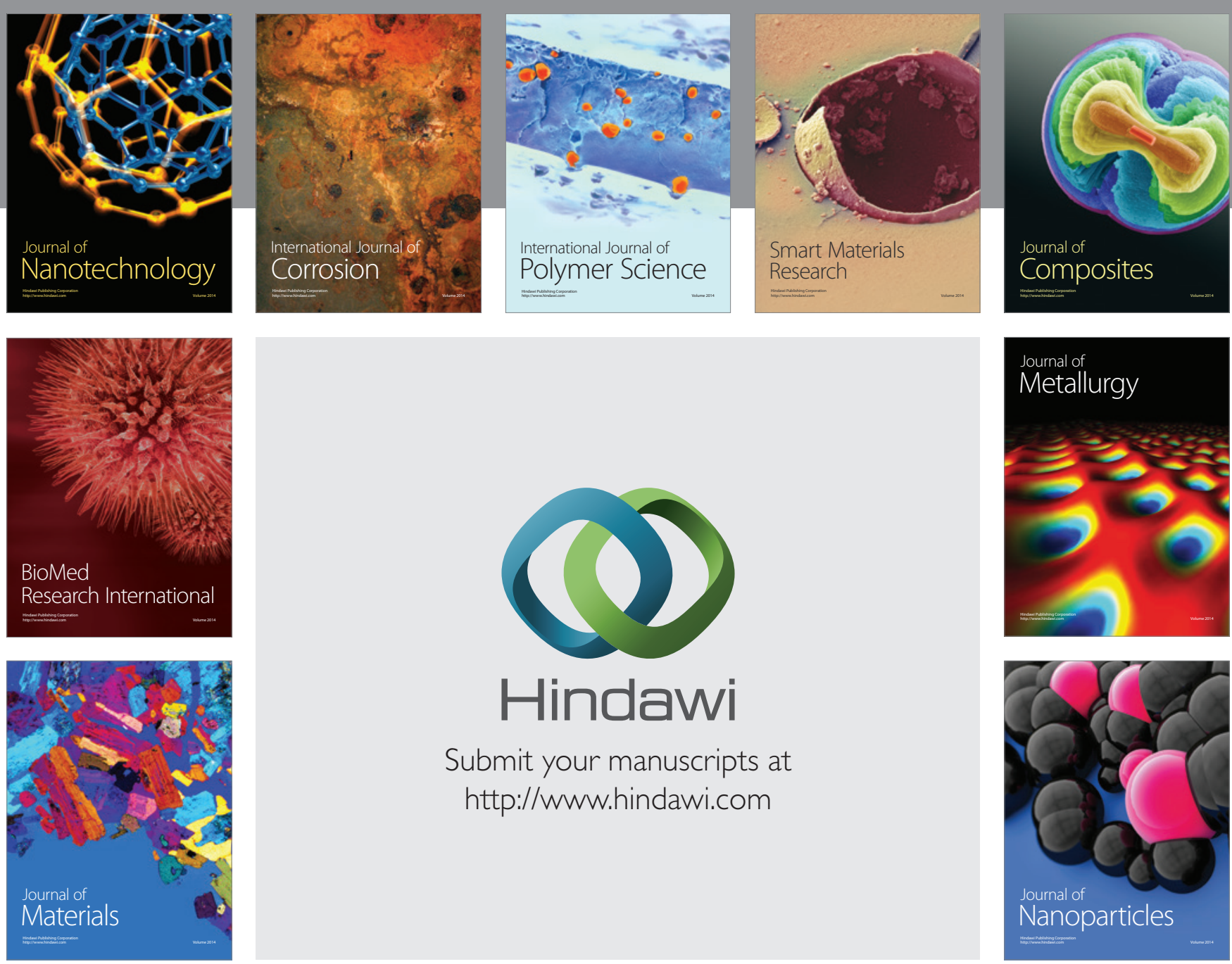

Submit your manuscripts at http://www.hindawi.com
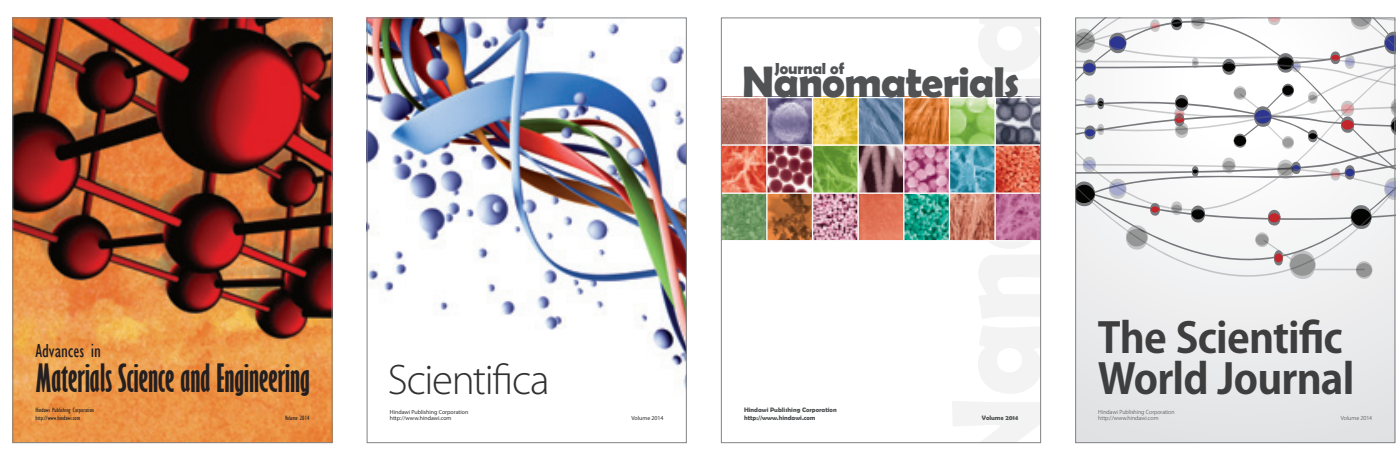

\section{The Scientific World Journal}
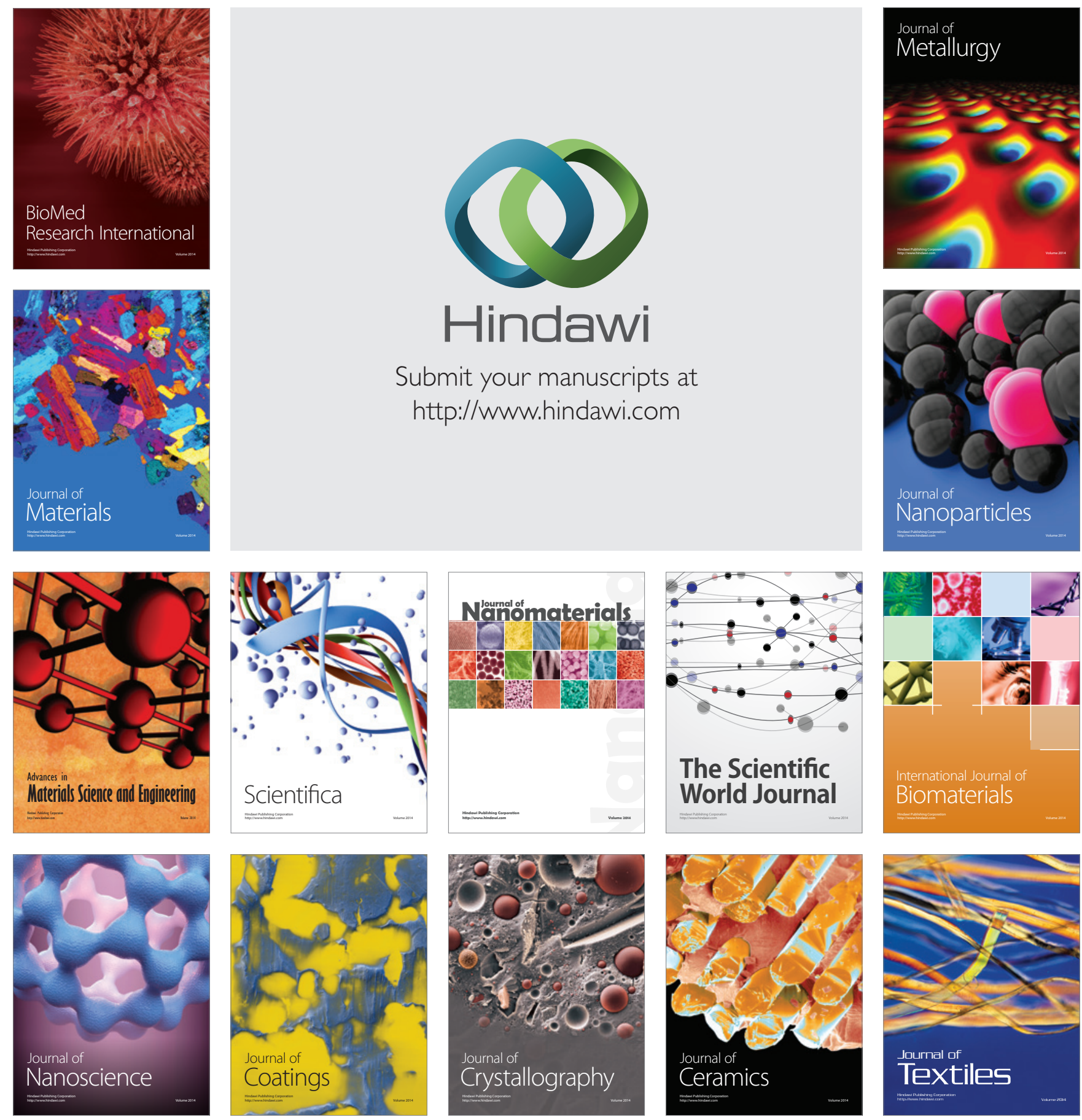